Volume 8, No.3, May - June 2019

International Journal of Advanced Trends in Computer Science and Engineering

Available Online at http://www.warse.org/IJATCSE/static/pdf/file/ijatcse93832019.pdf

https://doi.org/10.30534/ijatcse/2019/93832019

\title{
Simulation and Visualization of Acoustic Underwater Sensor Networks using Aqua-Sim and Aqua-3D : An Evaluation
}

\author{
Nasarudin Ismail ${ }^{1}$, Mohd Murtadha Mohamad ${ }^{2}$, Mohamad Aizi Salamat ${ }^{3}$ \\ ${ }^{1}$ Pusat Teknologi Maklumat, Universiti Tun Hussein Onn Malaysia, 86400 Parit Raja, Batu Pahat, Johor, Malaysia, \\ nasar@uthm.my \\ ${ }^{2}$ School of Computing, Faculty of Engineering, Universiti Teknologi Malaysia, 81310 UTM Skudai, Johor, Malaysia, \\ murtadha@utm.my \\ ${ }^{3}$ Software Engineering Department, Faculty of Computer Science and Information Technology, Universiti Tun Hussein \\ Onn Malaysia, 86400 Parit Raja, Batu Pahat, Johor, Malaysia, \\ aizi@uthm.edu.my
}

\begin{abstract}
Contrasting the Radio Frequency (RF) or Electro Magnetic (EM) wave based terrestrial network communication technologies, acoustic communication on Underwater Sensor Networks (UWSNs) add a unique set of challenges due their long propagation delays, low data rates, and high transmit power. Due to characteristic of basic acoustic nature and hence require an entire redesign in all the layer of network protocol stack. Most importantly, the current established network technology that were developed for conventional terrestrial wireless sensor networks (TWSNs) are not suitable for directly applying them in underwater scenarios. Due to this reason, the research in network technology of UWSNs has been underway to overcome the lack of available network technology in UWSNs. In this work, we are going to study some of the available simulators for simulating a UWSNs environment and enlighten the reasons for selecting Aqua-Sim and Aqua-3D for our underwater network simulations. In this research, the performance of the basic routing protocols in AquaSim such as Underwater Flooding (UWFlooding), VectorBased Forwarding (VBF), and Hop-by-Hop Vector-Based Forwarding Protocol (HH-VBF) is evaluated with the metrics packet delivery ratio (PDR), cumulative delay, throughput and energy consumption under dense network environment. The result of our study showed that VBF and $\mathrm{HH}-\mathrm{VBF}$ are performed better in terms of PDR, cumulative delay and energy consumption, while UWFlooding is performed better in terms of cumulative delay.
\end{abstract}

Key words: UWSNs, Aqua-Sim. Aqua-3D, Routing Protocol, Flooding, Underwater Sensor Networks (UWSNs)

\section{INTRODUCTION}

Currently, human knowledge about underwater environment is still scratching the surface in comparison with land exploration. Exploration of knowledge about the land and its structure are remarkably a success due to the technological advancement in Wireless Sensors Network (WSN)[1]. As such, researchers are eagerly wanted to explore in an underwater environment with the same technology which can be called as Underwater Sensor Networks (UWSNs) [2]. However, due to its unique features in underwater environments such as high water pressure, wide area and environmentally vicious conditions, the use of UWSN is the most appropriate way by using un-manned discovery in that environment [3].

In line for the unique characteristic of underwater environment, there are several issues or challenges when talking about designing communication network in the underwater environment especially in designing the routing protocol [4] and MAC protocol [5].

First, the deployment area in UWSNs which is using 3 dimensional architecture is so large. However due to the underwater sensor node is very expensive compared to the TWSNs sensor, the deployment of nodes is usually in sparse and the sensor nodes are suffered in the water current movement [6].

Second, UWSNs sensor nodes are powered by battery; instead TWSNs sensor nodes use solar to extend power. In UWSNs nodes are solely powered by battery which cannot be recharged and difficult to be replaced due to water conditions [2]. This is the reason why designing energy efficiency routing is so important in UWSNs.

Third, by using the acoustic signal as a medium of communication, UWSNs is prone to long propagation delay, high path loss, limited bandwidth and high energy consumption compared to the radio signals used in TWSNs [3,7-9]. Finally, due to the inapplicable use of Global Position System (GPS) in UWSNs since the high radio frequency employed by GPS is rapidly absorbed in underwater environment, the procedure of placing and obtaining the location information of sensor nodes become very difficult in UWSNs compared to TWSNs $[3,10]$.

In wireless sensor network environment especially on underwater, to validate and verify an algorithm or protocol must use the simulation tools instead of actual deployment. In UWSNs research, to use the real environment to recurrence the test and to verify the effects of the iterations are so expensive and risky mission. Therefore, most of the research events for the UWSNs need to be simulated efficiently. 
In this paper, a several simulation tools that were useful to simulate UWSNs is studied and each of the features offered by them also being compared. Both of the open source and commercial simulation is being selected in this research, we compare the results and provide advantages in the implementation and problems faced. So, researchers have a choice to choose the simulation tools that are appropriate for their work in studied UWSNs.

The rest of this paper is organized as follows. Section 2 provides the description of various simulation tools and reason why we choose Aqua-Sim and Aqua3d. Section 3 includes the simulation setup use in Aqua-Sim and Aqua3D. Section 4 includes the discussion and results of the simulation experiments and last section is conclusion for future research works.

\section{SIMULATORS AVAILABLE FOR SIMULATING ACOUSTIC UNDERWATER NETWORK}

As of now, there is no standard simulation/emulator to cater for all characteristics of UWSNs like acoustic communication, propagation delay, $3 \mathrm{~d}$ deployment etc. Most popular network simulators such as QualNet, OPNET, Aqua-Glomo, SUNSET, NS-2 and NS-3 cannot effectively reproduce the actual underwater environment in their simulation tools [11].

\subsection{Underwater Network simulation under QualNet Simulator}

QualNet is a one of the popular simulation tool for network enviroment that provides a tool for designing testing and training of network behavior for any type of communications network. It has a GUI that is very good with many choices for designing networks. QualNet is regarded as state of the art simulator for designing large heterogeneous networks. The main modules offered by QualNet are as follows QualNet Architect - acts as visualization tool, QualNet Analyzer - covers for statistical tools for network performance analyze, QualNet packet tracer - GUI analyzer packet tracer, QualNet File Editor Editing tool and the last one QualNet command line - to provide command line interfaces.

QualNet provides a flexible platform to design and test a network protocol. It is used to support for parallel processing. It is also good to study the localization-related because it supports both Geographic and Cartesian coordinate systems. However in wireless network situation it only supports Random-waypoint mobility model and for others, need to design by the users. As we know that QualNet is design specifically for both wired and wireless radio networks. So majority of the built-in channel properties, protocols, network components, and deployment models are specific to radio networks. Therefore to do a experiment of UWSNs at QualNet, we need to recreate the model of acoustic features and channel properties of UWSNs, which is a difficult task.

\subsection{Underwater Network simulation under OPNET Simulator}

OPNET is an advanced event-driven network simulator tool that runs at the packet level. It can be used for network design or analysis and purposely for research. In OPNET, the modelling can be separated into three domains networks, nodes, and processes. Analysis and debugging in OPNET is using GUI based environment. Although OPNET is one of the leading network modeling and simulation environment, but in order to simulate the underwater environment, we need to convert 3 stage from the 14 pipeline stages in OPNET. It is necessary to modify the 6 stage to include propagation delay of acoustic wave, the 8 stage to include a receiver power for sensor and the 10 stage to include the background noise to the defined underwater acoustic channel model simulation [12]. However, OPNET is a commercial software with license for usage and because of that it is only suitable for industrial standard and real-time usage.

\subsection{Underwater Network simulation under Aqua- Glomo Simulator}

Aqua-Glomo is GloMoSim (Global System for Mobile Information System Simulator) upgraded version, was developed for the large-scale communication network simulator. The physical and network layer packages of GLOMOSIM are improved to build a package that is suitable for UWSNs. PARSEC (parallel complex systems Simulation Environment) implementation is based on $\mathrm{C}$ language. Acoustic communication model is applied at physical layer, Thorp attenuation model used is a model which depends on the distance and frequency. RMTG (geo-broadcast based on routing and multicast trees) is applied in network layer, which is a geolocation protocol developed precisely for UWSNs. In Aqua-Glomo only Random-Waypoint mobility model is used. However, Aqua-Glomo was developed to study the physical and network layer only and it is still lack of some features needed for underwater acoustic communication especially on simulation model and mathematical model for acoustic communication.

\subsection{Underwater Network simulation under NS-3 Simulator}

Just like NS-2, this NS-3 is also an open source discrete event network simulator tool. It is developed using Python and $\mathrm{C}++$ with scripts and provides visualization simulation [13]. However this new NS-3 tool is not back compatible with previous NS-2 code. For UWSNs scenarios, NS-3 come with Underwater Acoustics Networks(UAN) framework [13] which allows modelling of UWSNs. The UAN framework has four divisions: channel model, PHY, MAC and AUV. The UAN propagation model makes it easy for users to select matching acoustics and suitable attenuation models. In UAN framework, there are three deployment models is supported, which are Ideal Channel models, Thorp models, and Bellhop models. Zero path loss is considers in Ideal Channel model. To calculate the path loss, Thorp attenuation model is used. While for the last model, Bellhop, it used to gathers the propagation 
information from the database. For UAN PHY model, it has several PHY class, like UanPhyGen, which is responsible for packet acquisition, determining errors and successful package delivery. While for Dual PHY layer, UanPhyDual, which wraps two generic PHY layers together can be used to emulate devices that include two recipients. In Autonomous Vehicle Underwater (AUV) UAN, it allows users to program AUV to navigate the route and controlling several AUV parameters such as direction, velocity, depth, and field.

Additionally to the standard visualization tools, alternative visualization package called Netanim was developed that integrates with the NS-3 package itself. To analyse trace files, user would use software called Tracemetrics. In the NS-3 simulation, you can generate .pcap files and import them into the familiar packet analyser software wireshark.

\subsection{Underwater Network simulation under SUNSET Simulator}

Another simulator tool based from NS-2, call as Sapienza University Networking framework for underwater Simulation, Emulation and real-life Testing(SUNSET) can be used for UWSNs[14]. It was developed by the Department of Information in University of La Sapienza based on underwater characteristic. SUNSET would use different channel models during the network simulation. SUNSET can be connected to real external hardware for real test bed in emulation mode. There are several benefits to use SUNSET, such as it can be a real-time scheduler, can use several threads in emulation mode, and also enhanced the interference model. The timing module in SUNSET could provide an accurate information about sensors and packet communication delays, which are usually not well-thought-out during the simulation. It has universal and application driver modules that incorporate with sensors, acoustic modems and AUVs. However SUNSET creates an overhead on limited resource for underwater systems by which each of the node needs to run an instance of NS-2 during the simulation.

\subsection{About the Simulator of our Choice Aqua-Sim and Aqua3D}

During our previous study [4] we realized that Aqua-Sim is the only available open source simulation tool which will accommodate most of the aspects of a typical UWSN. Mostly among them all, Aqua3D of the Aquasim project is the only available tool that can be used to visualize a typical 3D underwater network and its transmissions. So, in this work, we decided to use Aquasim and Aqua3D for our simulation experiments and visualization.

\subsubsection{Network Simulator 2 (NS-2)}

NS-2 is a discrete event simulator targeted at networking research. NS-2 provides substantial support for simulation of TCP, routing, and multicast protocols over wired and wireless networks. NS2 is the second version of NS (Network Simulator). NS is originally based on REAL network simulator [REAL]. The first version of NS was developed in 1989 and evolved a lot over the past few years. The current NS project is supported through DARPA. The current second version NS2 is widely used in academic research and it has a lot of packages contributed by different non-benefit groups.

\subsubsection{Aqua-Sim[15]}

Aqua-Sim is a NS-2 based simulator for simulating UWSNs environment. It can simulate acoustic signal attenuation, packet collisions, routing in UWSNs. AquaSim supports three-dimensional deployment. Further, Aqua-Sim can easily be integrated with the existing codes in NS-2. Aqua-Sim is in parallel with the CMU wireless simulation package. Basically, Aqua-Sim is a NS-2 based network simulator that is able to simulate UWSNs efficiently. Aqua-Sim is nothing but an enhanced or extended version of NS-2. Aqua-Sim is free from wireless simulation packages and is not affected by any change in the wireless packages. On the other hand, any change to Aqua-Sim is also confined to itself and does not have any impact on other packages in NS-2. In this way, Aqua-Sim can evolve independently.

In Aqua-Sim, network entities are implemented as classes to follows object-oriented design style. Aqua-Sim effectively simulates the attenuation of underwater acoustic channels and the collision behaviours in long delay acoustic networks. Moreover, Aqua-Sim supports threedimensional network deployment and provides a rich set of basic and advanced protocols. Through several case studies, the authors of [15] showed that Aqua-Sim is a powerful simulation tool, with high fidelity and flexibility, for underwater networking research. Have presented the design and implementation of Aqua-Sim, a packet level underwater sensor network simulator based on NS-2. The following class diagram in Figure 1 explains its architecture and design.

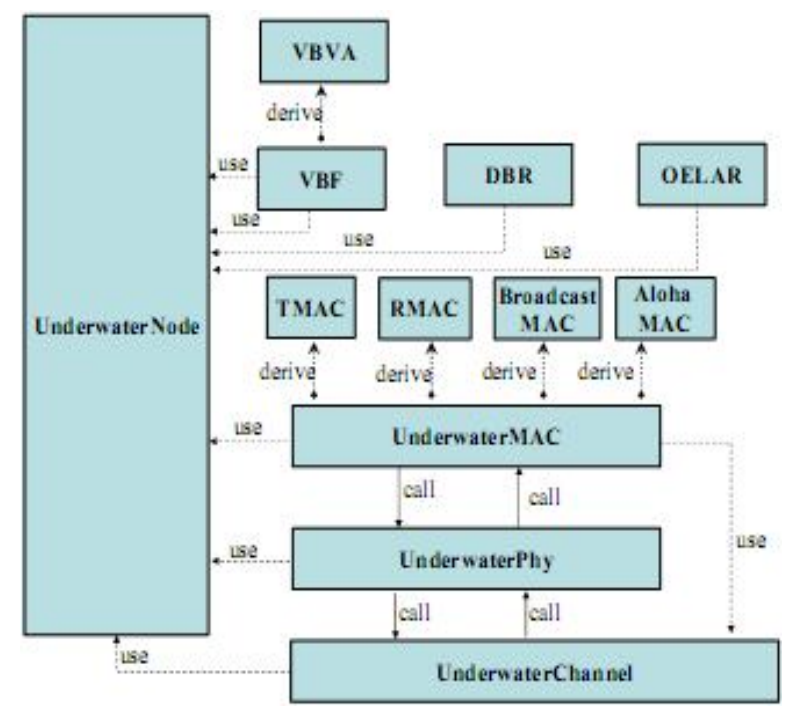

Figure 1: Class diagram of Aqua-Sim 


\subsubsection{Aqua-3D}

Aqua-3D is being developed at the University of Connecticut to be the counterpart to the UWSNs AquaSim. Aqua-3D is an enhanced Network Animator (NAM) of NS-2. In fact, Aqua-3Ds extends the functionality of NAM to make it potential to visualize the threedimensional UWSNs. Aqua-3D is capable of rendering simulations in 3D graphics, a fully controllable camera that can be viewed in 360 degrees. It has adjustments option, change appearance, save different camera angles, show lines for node transfer paths, show network topology, and options to save some interesting events. Aqua-3D had the ability to visualize correctly the trace files in UWSNs simulation. Figure 2 shows the example visual Aqua-3D GUI.

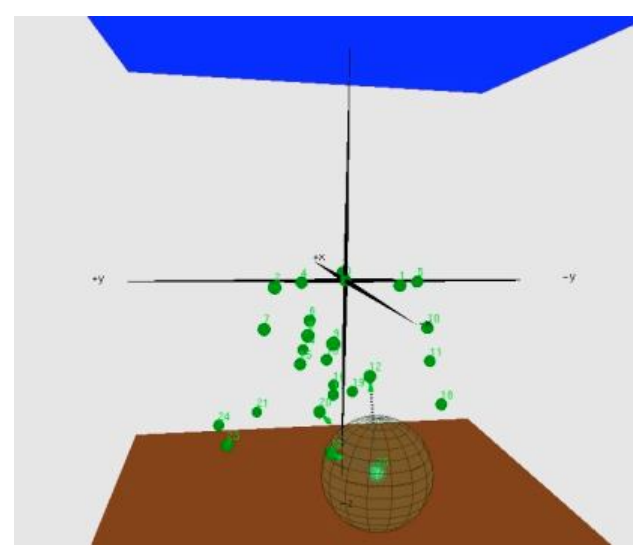

Figure 2: Aqua3D GUI Example

\section{SIMULATION OF ACOUSTIC UNDERWATER SENSOR NETWORKS USING AQUA-SIM AND AQUA-3D}

\subsection{Simulation Setup}

The underwater sensor nodes are placed at random location and some selected set of underwater sensor nodes would start CBR traffic and send packets to the one sink node located at the surface of the water. Most of the parameters of the simulations were used as default of Aqua-Sim. There are three routing protocols used in this simulation, UWFlooding, Vector-Based Forwarding (VBF) [16] and Hop by Hop-Vector Based Forwarding (HH-VBF) [17]. While for some of the important parameters that were used in this simulation are given in the following Table 1 . The simulations were repeated for several times and only the average values of results are taken into account. The randomness of the topology and network conditions, traffic start and stop time were controlled by seeding the random number generator with respect to the run number.
Table 1: Some Important Parameters of the Simulation

\begin{tabular}{|l|l|}
\hline Parameters & Value \\
\hline Area & $500 \mathrm{~m} * 500 \mathrm{~m} * 500 \mathrm{~m}$ \\
\hline Channel & Acoustic Waves \\
\hline $\begin{array}{l}\text { No Underwater } \\
\text { Nodes }\end{array}$ & 100 \\
\hline Number of Sinks & One at the Surface \\
\hline Movement Model & Static Model \\
\hline Routing Protocols & $\begin{array}{l}\text { UWFlooding, VBF, HH- } \\
\text { VBF }\end{array}$ \\
\hline Propagation Model & UnderwaterPropagation \\
\hline Physical Model & UnderwaterPhy \\
\hline Mac Protocol & Broadcast Mac \\
\hline Traffic Type & CBR \\
\hline CBR Flows & $1,2,3,4,5,6$ \\
\hline Initial Energy Nodes & $1000 \mathrm{~J}$ \\
\hline Transmission Range & $250 \mathrm{~m}$ \\
\hline Energy Consumption & $\begin{array}{l}\mathrm{P}_{\mathrm{tx}}=2 \mathrm{~W}, \mathrm{P}_{\mathrm{rx}}=0.1 \mathrm{~W}, \mathrm{P}_{\text {idle }}= \\
10 \mathrm{~mW}\end{array}$ \\
\hline Geometric Spreading & $2(\mathrm{Spherical})$ \\
\hline $\begin{array}{l}\text { Acoustic Signal } \\
\text { Speed }\end{array}$ & $1500 \mathrm{~m} / \mathrm{s}$ \\
\hline $\begin{array}{l}\text { Avg. Data Packet } \\
\text { Size }\end{array}$ & $<200 \mathrm{bytes}$ \\
\hline Data Transfer Rate & $10 \mathrm{kbps}$ \\
\hline Simulation Time & $500 \mathrm{~s}$ \\
\hline Runs & 5 \\
\hline
\end{tabular}

\subsection{Metrics Used for Evaluating the Performance}

In order to evaluate the performance of UWSN network routing protocols, the following metrics were considered:

Packet Delivery Ratio - PDR (\%): Packet delivery ratio is the ratio of the number of packets successfully received by the sink node at the surface of water to the total number of packets sent into the network by the UWSN sensor nodes.

Cumulative Delay (milliseconds): The average time interval between the generation of a packet in a source UWSN node and the successfully delivery of the packet at the UWSN sink node. It counts all possible delays that can occur in the source and all intermediate nodes, including queuing time, packet transmission and propagation, and retransmissions at the MAC layer. In this work, we are finding the average of the cumulative end to end delay of all the data packets. It is measured in milli-seconds.

Throughput (Kbps): The throughput metric measures how well the network can constantly provide data to the sink. Throughput is the number of bytes or bits arriving at the sink over time. It is generally measured in Kilobits per second or Mega Bits per second.

Energy Consumption (Jules): The average of the total consumed energy of all the nodes of the network. It is measured in Jules. 
4

\section{RESULTS AND DISCUSSION}

During this simulation, each data flow will try to send a maximum of 100 data packets. So if there will be 6 flows, then it will try to send 600 packets in total. To simulate somewhat high density scenario, the number of underwater sensor nodes is kept as 100 in all these experiments and keeping those 100 nodes in a small topographical area (500mx500mx500m) makes it as a little bit denser network. The Analysis was made with the default output $\log$ files that were exclusively generated by Aqua-Sim extension of the simulator.

\subsection{Analysis of the Results}

The following Figure 3 shows the performance in terms of PDR with respect to different number of traffic flows. As shown in the graph, the performance of UWFlooding is poor and even getting very poor with respect to the increase number of simultaneous traffic flows from different nodes. While for the VBF and $\mathrm{HH}-\mathrm{VBF}$ routing protocols, their performance seems to be almost equal and better than UWFlooding. However their performance in terms of PDR is getting worst with respect to the increase in the number of simultaneous data flows. This result shows that because of the increase traffic packet data in network would lead to more collision and drop packets.

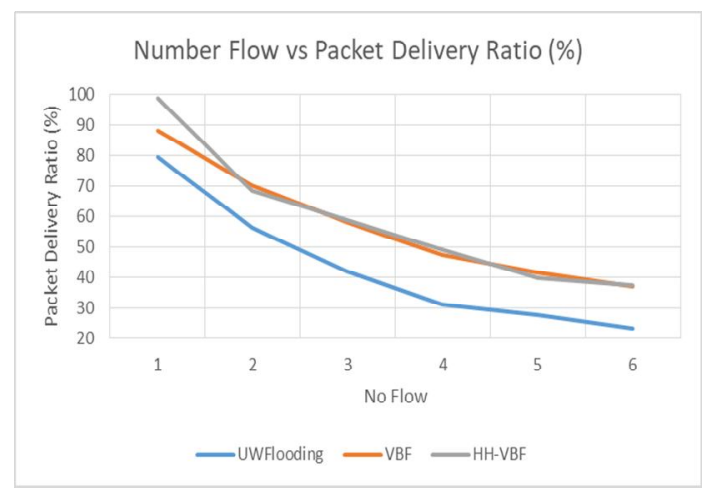

Figure 3: Number Flow vs Packet Delivery Ratio (\%)

The following Figure 4 shows the performance in terms of cumulative delay with respect to different number of traffic flows. The performance of the HH-VBF routing protocols seems to be little bit poor than that of normal VBF. While for the performance of UWFlooding in terms of end to end cumulative delay is better than the other two routing protocols and even almost constant irrespective of number of traffic flows.

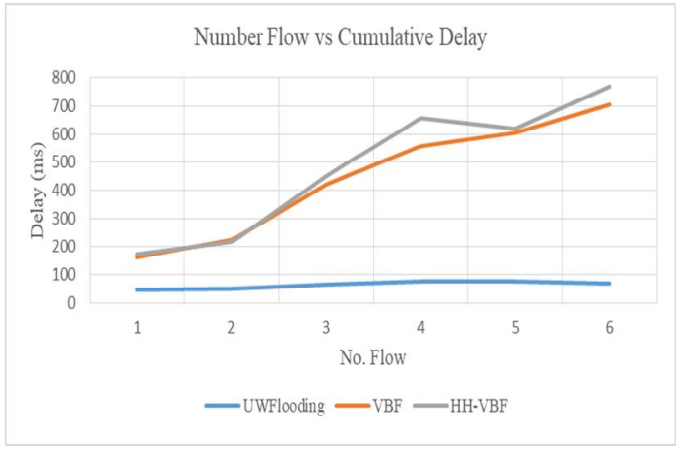

Figure 4: Number Flow vs Cumulative Delay

The following Figure 5 shows the throughput with respect to different number of traffic flows. As shown in the graph, the performance of UWFlooding is poor and even getting very poor with respect to the increase of number of simultaneous data flows from different UWSN nodes. The performance of the VBF and $\mathrm{HH}-\mathrm{VBF}$ routing protocols seems to be almost equal and giving acceptable increase in throughput with respect to the increase in the number of simultaneous number of traffic flows.

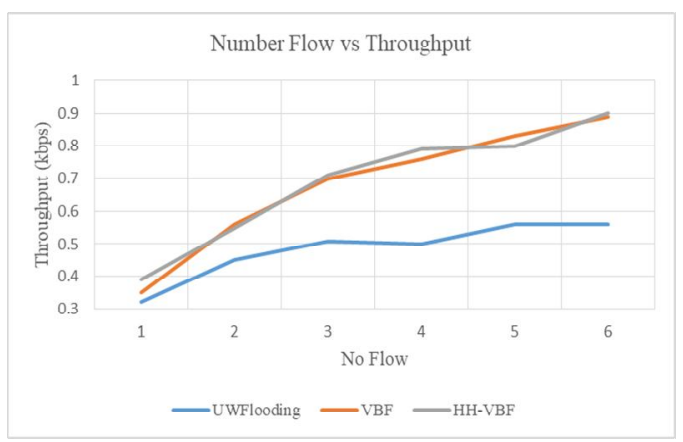

Figure 5: Number Flow vs Throughput

The following Figure 6 shows the performance in terms of energy consumption with respect to different number of traffic flows. The performance of the VBF and HH-VBF routing protocols seems to be equal each other and much better than that of UWFlooding routing protocol. As shown in the Figure 6, the performance of UWFlooding in terms of energy consumption is very poor with respect to increase number of traffic flows.

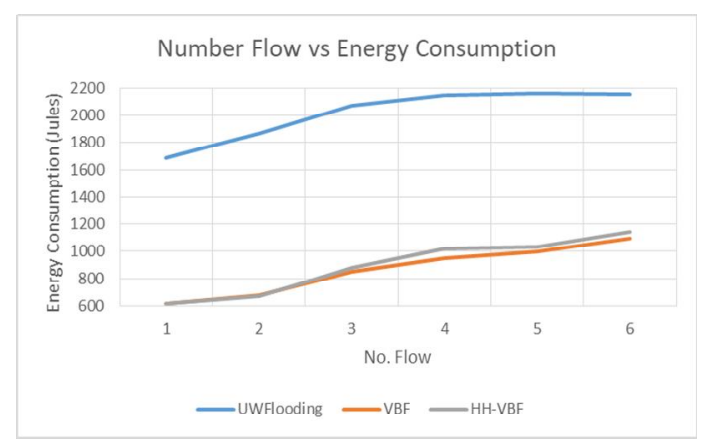

Figure 6: Number Flow vs Energy Consumption 
5

\section{CONCLUSION}

In this paper, we highlighted some of the good features of Aqua-Sim and Aqua-3D and used them for evaluating three default UWSNs routing protocols, UWFlooding, VBF and HH-VBF. The outputs in the above result sections of this paper clearly show the usability of AquaSim and Aqua-3D for doing a quality research on acoustic UWSNs and their routing protocols especially in network layer. This study shows that, performance of VBF and $\mathrm{HH}-$ VBF are better in terms of PDR, throughput and energy consumption. While UWFlooding only performs better in cumulative delay due to opportunistic routing method which employ the multipath technique.

In our future works, an extensive study and evaluation on the available routing protocols to improve some of the existing routing protocols by applying a multiple sink node instead of only one sink node to further study the effect on dense network scenarios in UWSNs.

\section{ACKNOWLEDGEMENT}

This work is supported by Universiti Tun Hussein Onn Malaysia under Multidisciplinary Research (MDR) Grant no vote U899 and Universiti Teknologi Malaysia.

\section{REFERENCES}

[1] M.S. Devi, P. Kumar, Wireless sensor Network based Industrial Automation using Internet of Things (IoT), Int. J. Adv. Trends Comput. Sci. Eng. $\quad 7 \quad$ (2018) 92-86. doi:10.30534/ijatcse/2018/01762018.

[2] I.F. Akyildiz, D. Pompili, T. Melodia, Underwater acoustic sensor networks: Research challenges, Ad Hoc Networks. 3 (2005) 257-279. doi:10.1016/j.adhoc.2005.01.004.

[3] M. Ayaz, I. Baig, A. Abdullah, I. Faye, A survey on routing techniques in underwater wireless sensor networks, J. Netw. Comput. Appl. 34 (2011) 1908-1927. doi:10.1016/j.jnca.2011.06.009.

[4] N. Ismail, M.M. Mohamad, REVIEW ON ENERGY EFFICIENT OPPORTUNISTIC ROUTING PROTOCOL FOR UNDERWATER WIRELESS SENSOR NETWORKS, KSII Trans. Internet Inf. Syst. 12 (2018) 3064-3094. doi:10.3837/tiis.2018.07.006.

[5] S. Choi, S. Kim, Multi-Channel MAC Protocol based on Dynamic Time Slot Allocation for Underwater Sensor Networks, Int. J. Adv. Trends Comput. Sci. Eng. 8 (2019) 187-193. doi:10.30534/ijatcse/2019/13822019.
[6] I.F. Akyildiz, D. Pompili, T. Melodia, Challenges for efficient communication in underwater acoustic sensor networks, ACM SIGBED Rev. 1 (2004) 38. doi:10.1145/1121776.1121779.

[7] N.Z. Zenia, M. Aseeri, M.R. Ahmed, Z.I. Chowdhury, M. Shamim Kaiser, Energy-efficiency and reliability in MAC and routing protocols for underwater wireless sensor network: A survey, J. Netw. Comput. Appl. 71 (2016) 72-85. doi:10.1016/j.jnca.2016.06.005.

[8] M. Ahmed, M. Salleh, M.I. Channa, Routing protocols based on node mobility for Underwater Wireless Sensor Network (UWSN): A survey, J. Netw. Comput. Appl. (2016) 1-11. doi:10.1016/j.jnca.2016.10.022.

[9] D. Pompili, I.F. Akyildiz, Overview of Networking Protocols for Underwater Wireless Communications, IEEE Commun. Mag. 47 (2009) 97-102. doi:10.1109/MCOM.2009.4752684.

[10] T. Melodia, H. Kulhandjian, L. Kuo, E. Demirors, Advances in Underwater Acoustic, in: Mob. Ad Hoc Netw. Cut. Edge Dir. Second Ed., 2013: pp. 804-854.

[11] A.P. Das, S.M. Thampi, Simulation Tools for Underwater Sensor Networks: A Survey, Netw. $\begin{array}{llll}\text { Protoc. Algorithms. } 8 \text { (2017) } 41 . & \end{array}$ doi:10.5296/npa.v8i4.10471.

[12] C. Wang, Y.J. Fang, Channel model simulation for underwater acoustic sensor networks using OPNET, Int. Conf. Commun. Technol. Proceedings, ICCT. 0 (2010) 141-144. doi:10.1109/ICCT.2010.5689314.

[13] UAN Framework, (n.d.). https://www.nsnam.org/docs/models/html/uan.html (accessed February 13, 2019).

[14] C. Petrioli, R. Petroccia, D. Informatica, SUNSET: Simulation, emulation and real-life testing of underwater wireless sensor networks, Proc. IEEE UComms 2012. (2012) 12-19. doi: $10.1145 / 2532378.2532424$.

[15] P.X.P. Xie, Z.Z.Z. Zhou, Z.P.Z. Peng, H.Y.H. Yan, T.H.T. Hu, J.-H.C.J.-H. Cui, Z.S.Z. Shi, Y.F.Y. Fei, S.Z.S. Zhou, Aqua-Sim: An NS-2 based simulator for underwater sensor networks, Ocean. 2009, MTS/IEEE Biloxi - Mar. Technol. Our Futur. Glob. Local Challenges. (2009) 1-7.

[16] P. Xie, J.-H. Cui, L. Lao, VBF: Vector-Based Forwarding Protocol for Underwater Sensor Networks, in: Lect. Notes Comput. Sci. (Including Subser. Lect. Notes Artif. Intell. Lect. Notes Bioinformatics), 2006: pp. 1216-1221. doi:10.1007/11753810_111.

[17] N. Nicolaou, A. See, P. Xie, J.-H. Cui, D. Maggiorini, Improving the Robustness of Location-Based Routing for Underwater Sensor Networks, in: Ocean. 2007 - Eur., IEEE, 2007: pp. 1-6. doi:10.1109/OCEANSE.2007.4302470. 